\title{
Detection of multivessel coronary artery disease: looking beyond the extent of perfusion abnormalities
}

\author{
Dipan Desai, DO, Gorgi Kozeski, DO, and Olakunle Akinboboye, MD
}

\section{See related article,}

\section{doi: 10.1007/s12350-008-9003-7}

The sensitivity of stress myocardial perfusion scintigraphy for detecting coronary artery disease (CAD) in patients with multivessel CAD is high. ${ }^{1-4}$ However, its ability to predict the presence of multivessel disease is limited. It has been suggested that the presence of left main or multivessel disease is missed in up to $50 \%$ of cases. ${ }^{5,6}$ The possible reasons for this include balanced perfusion abnormality, where the absence of a normal reference segment limits sensitivity, early plateau of tracer uptake, which limits detection of borderline stenoses, and early stoppage of exercise as a result of symptoms or signs due to the severest lesion.

There is evidence that ancillary clinical, exercise, and scan variables can improve the sensitivity of a gated SPECT myocardial perfusion study for detection of multivessel CAD. ${ }^{7}$ These include poor exercise tolerance and development of chest pain and/or electrocardiographic evidence of ischemia at a low exercise workload, ${ }^{8}$ increased lung/heart tracer uptake ratio, ${ }^{9}$ and transient ischemic dilation of the left ventricle following stress (TID), which was shown to be a specific but fairly insensitive marker of multivessel CAD. Initial studies with planar Tl-201 imaging found a cut-off TID ratio of 1.12 to have the best sensitivity and specificity for detecting multivessel CAD. A subsequent study with dual-isotope imaging reported an optimal cut-off of 1.22. ${ }^{10}$ Other approaches include integration of functional assessment by gated SPECT with conventional perfusion analysis; ; 11 comparison of RV to LV activity, which was shown to be a marker of severe CAD,

From the New York Hospital Medical Center of Queens, Weill Medical College of Cornell University, Queens, NY.

Reprint requests: Olakunle Akinboboye, MD, New York Hospital Medical Center of Queens, Weill Medical College of Cornell University, Queens, NY; olak2@yahoo.com.

J Nucl Cardiol 2009;16:4-5

$1071-3581 / \$ 34.00$

Copyright $(2008$ by the American Society of Nuclear Cardiology. doi:10.1007/s12350-008-9036-y particularly high-grade left main or proximal LAD or LCx, without concomitant proximal RCA disease; ${ }^{12}$ and normalization of the stress to a normal reference segment identified on the resting study. ${ }^{13}$

Others studies have suggested that a better approach for detecting multivessel CAD is to perform PET perfusion imaging. ${ }^{14}$ The advantages of PET include the availability of a robust attenuation correction algorithm and the fact that some PET tracers have superior flow tracking ability to the commonly used technetium-based SPECT perfusion tracers. However, the limited availability of PET scanners limits the clinical utility of this approach.

Hida et $\mathrm{al}^{15}$ evaluated the use of left ventricular function after exercise as a predictor of multivessel CAD in 175 patients who underwent gated SPECT myocardial perfusion imaging and coronary angiography within 3 months. They reported sensitivities and specificities for detecting multivessel CAD of $66 \%$ and $87 \%, 52 \%$ and $83 \%$, and $46 \%$ and $90 \%$ for an increase in post-exercise ESV of $5 \mathrm{~mL}$, decrease in post-exercise ejection fraction of $5 \%$, and a summed difference score of 9, respectively. On multivariate analysis, they found that the combination of post-stress increase in ESV, summed difference score, and diabetes mellitus had the best sensitivity and specificity (72\% and $84 \%$, respectively) for detecting multivessel CAD. In this issue of the journal, ${ }^{16}$ the same investigators reported on the diagnostic value of post-stress left ventricular volume and function analyses with vasodilator stress testing. They studied 119 patients who underwent gated Tc-sestamibi gated SPECT with adenosine triphosphate and coronary angiography within 3 months. Fifty-one percent of the patients had multivessel CAD including 45 patients with double-vessel and 16 patients with triplevessel disease. They found sensitivities and specificities for detecting multivessel CAD of $57 \%$, and $64 \%$ for summed stress core $\geq 14$, and $53 \%$ and $88 \%$ for summed difference score $\geq 9$. These sensitivities, specificities, and accuracy measurement after ATP loading were similar to their observations with exercise stress. In addition, the authors evaluated the utility of change in end-systolic volume, end-diastolic volume, and ejection fraction and stress-induced volume ratio (SIVR) defined 
as stress to rest ratios of (ESV $\times 5+\mathrm{EDV})$ for detecting multivessel CAD. They found that a derived cut-off of $>6 \mathrm{~mL}$ increase in ESV or EDV, or a $>5 \%$ decrease in EF after stress, and SIVR of $\geq 1.13$ demonstrated sensitivities of $59 \%, 80 \%, 61 \%$, and $74 \%$, respectively, and specificities of $72 \%, 76 \%, 77 \%$, and $78 \%$ respectively. On multivariate analysis, the combination of post-stress increase in ESV and the SDS performed best for identification of multivessel CAD with $80 \%$ sensitivity and $76 \%$ specificity.

Identifying multivessel disease pattern on a SPECT myocardial perfusion study is very important because of its management implications. Patients with multivessel disease have poorer prognoses and are more likely to benefit from coronary revascularization.

Hida et $\mathrm{al}^{16}$ offered an approach to detection of multivessel CAD by gated SPECT, which integrates the extent of reversible perfusion abnormality and poststress LV dilation. The strength of this approach is that it uses data that are readily available on a standard gated SPECT myocardial perfusion study without requirement for additional processing. However, since increased SDS or post-stress LV dimensions are more likely to occur in patients with reversible CAD, it will be interesting to know how well the approach performs in patients with prior infarction.

Reliable detection of multivessel CAD remains a challenge for gated SPECT perfusion imaging. Integration of information about the extent of reversible perfusion abnormality and change in left ventricular systolic volume post-exercise as suggested by Hida et $\mathrm{al}^{16}$ may mitigate this problem.

\section{References}

1. Sharir T, Bacher-Stier C, Dhar S, et al. Identification of severe and extensive coronary artery disease by postexercise regional wall motion abnormalities in Tc-99m sestamibi gated single-photon emission computed tomography. Am J Cardiol 2000;86:1171-5.

2. Yamagishi H, Shirai N, Yoshiyama M, et al. Incremental value of left ventricular ejection fraction for detection of multivessel coronary artery disease in exercise (201) Tl gated myocardial perfusion imaging. J Nucl Med 2002;43:131-9.

3. Lima RS, Watson DD, Goode AR, et al. Incremental value of combined perfusion and function over perfusion alone by gated SPECT myocardial perfusion imaging for detection of severe three-vessel coronary artery disease. J Am Coll Cardiol 2003;42:64-70.

4. Taillefer R, DePuey EG, Udelson JE, Beller GA, Latour Y, Reeves F. Comparative diagnostic accuracy of Tl-201 and Tc-99m sestamibi SPECT imaging (perfusion and ECG-gated SPECT) in detecting coronary artery disease in women. J Am Coll Cardiol 1997;29:69-77.

5. Rehn T, Griffith LS, Achuff SC, et al. Exercise thallium-201 myocardial imaging in left main coronary artery disease: sensitive but not specific. Am J Cardiol 1981;48:217-23.

6. Christian TF, Miller TD, Bailey KR, Gibbons RJ. Noninvasive identification of severe coronary artery disease using exercise tomographic thallium-201 imaging. Am J Cardiol 1992;70:14-20.

7. Iskandrian AS. Myocardial ischemia during pharmacological stress testing. Circulation. 1993;87:1415-7.

8. Hachamovitch R, Berman DS, Kiat H, et al. Exercise myocardial perfusion SPECT in patients without known coronary artery disease: incremental prognostic value and use in risk stratification. Circulation 1996;93:905-14.

9. Boucher CA, Zir LM, Beller GA, et al. Increased lung uptake of thallium-201 during exercise myocardial imaging: Clinical, hemodynamic and angiographic implications in patients with coronary artery disease. Am J Cardiol 1980;46:189-96.

10. Mazzanti M, Germano G, Kiat H, et al. Identification of severe and extensive coronary artery disease by automatic measurement of transient ischemic dilation of the left ventricle in dual-isotope myocardial perfusion SPECT. J Am Coll Cardiol 1996;27:161220.

11. Shirai N, Yamagishi H, Yoshiyama M, et al. Incremental value of assessment of regional wall motion for detection of multivessel coronary artery disease in exercise (201)Tl gated myocardial perfusion imaging. J Nucl Med 2002;43:443-50.

12. Williams KA, Schneider CM. Increased stress right ventricular activity on dual isotope perfusion SPECT: A sign of multivessel and/or left main coronary artery disease. J Am Coll Cardiol 1999;34:420-7.

13. Williams KA, Schuster RA, Williams KA Jr, Schneider CM, Pokharna HK. Correct spatial normalization of myocardial perfusion SPECT improves detection of multivessel coronary artery disease. J Nucl Cardiol 2003;10:353-60.

14. Bateman TM, Heller GV, McGhie AI, et al. Diagnostic accuracy of rest/stress ECG-gated Rb-82 myocardial perfusion PET: Comparison with ECG-gated Tc-99 $\mathrm{m}$ sestamibi SPECT. J Nucl Cardiol 2006;13:24-33.

15. Hida S, Chikamori T, Tanaka H, et al. Diagnostic value of left ventricular function after stress and at rest in the detection of multivessel coronary artery disease as assessed by electrocardiogram-gated SPECT. J Nucl Cardiol 2007;14:68-74.

16. Hida S. Diagnostic value of left ventricular function after adenosine triphosphate loading and at rest in the detection of multivessel coronary artery disease using myocardial perfusion imaging. J Nucl Cardiol 2008;15. 